\title{
Lewis Acids in Organic Synthesis. Approach to a Selection Strategy for Screening Experiments
}

\author{
Rolf Carlson, Torbjörn Lundstedt, Åke Nordahl and Michal Prochazka
}

Department of Organic Chemistry, University of Umeå, S-901 87 Umeå, Sweden

\begin{abstract}
Carlson, Rolf, Lundstedt, Torbjörn, Nordahl, Åke and Prochazka, Michal, 1986. Lewis Acids in Organic Synthesis. Approach to a Selection Strategy for Screening Experiments. - Acta Chem. Scand. B 40: 522-533.
\end{abstract}

A computer-assisted strategy for selecting suitable Lewis acid catalysts in organic synthesis is described. The strategy is based on principal components (PC) analysis of measured Lewis acid properties. A two-component model accounts for $65 \%$ of the variation in a set of 20 descriptors for 116 different Lewis acids. PCeigenvector projections of the data allow for a systematic search of test candidates in screening experiments. The strategy is demonstrated by experimental studies on 3 Lewis acid-catalyzed reactions: alkylation of silyl enol ether, Diels-Alder reaction, and Friedel-Crafts acylation.

Electrophilic catalysis by Lewis acids is essential in a number of important synthesis reactions. When a newly discovered reaction is to be elaborated into a useful synthetic procedure, an early and important step is to establish a suitable reaction system which can be optimized. With Lewis acid-catalyzed reactions, this implies the selection of suitable catalysts. However, this is not a trivial problem. With new reactions, mechanistic details are still obscure and it is not likely that such details will be revealed before the preparative utility of the reaction has been demonstrated. This means that a selection of catalysts for experimental studies will precede a detailed study of the reaction mechanism.

Although great effort has been spent on theoretical as well as empirical studies of Lewis acids, ${ }^{\prime}$ no general agreement has yet been reached on how to define "acidity" of Lewis acids. The rather fuzzy concept of hard/soft acids and bases further complicates the picture. ${ }^{2}$ This strongly indicates that it is not possible to use a single measured property as a probe of Lewis acidity. To achieve a satisfactory quantitative description, it is likely that several factors must be jointly considered and different multiparameter models have been suggested. ${ }^{1}$ However, these models depend on theoretical a priori assumptions which may or may not apply to the current synthetic problem. A modest but reasonable assumption is that Lewis acids, which are similar to each other with regard to various measured properties, may also show a similarity in behaviour when they take part in a chemical reaction. By this is meant that macroscopic properties are likely to be manifestations of intrinsic properties of the Lewis acids and that the same intrinsic properties are also responsible for the chemical reactivity. It certainly does not mean that chemical reactivity can be "explained" in a philosophical sense by the macroscopic properties. What is assumed is that macroscopic properties can be used as probes of intrinsic properties.

A number of physical and chemical properties can be used to characterize Lewis acids. Data on bulk properties and thermodynamic parameters can be compiled from various sources for a large number of acids. In the developmental phase of a new synthetic method it is not known with certainty which properties will be important for a catalytic effect. It is therefore desirable that a selection of test candidates for screening experiments cover a broad range of acid properties. However, it is impossible or at least very difficult to make such a selection from a huge table by mere inspection. In a recent paper $^{3}$ we discussed a similar problem - selection of solvents, and how a computer-assisted strategy based on multi- 
variate statistical analysis can be used to reduce the complexity of the problem. In this paper, we extend these principles to the selection of Lewis acids. The methods have been described in detail $^{3}$ so we do not repeat the discussion here.

\section{Results}

Initial study In an initial study, a set of 10 property descriptors for 28 Lewis acids, Table 1, was subjected to a principal components (PC) analysis. A two-component PC model was significant according to cross validation and accounted for $54 \%$ of the variation in the descriptors. The result is illustrated by the PC projection in Fig. 1 (a). The important point is that the systematic variation in the original data set can be described by only two descriptors $t_{1}$ and $t_{2}$. The extent to which each of the original descriptors contributes to the principal components $t_{1}$ and $t_{2}$ is shown in the loading plot in Fig. 1 (b).

The PC projection can be used to select test candidates for a screening experiment. A thorough discussion of various selection strategies is given in Ref. 3 . Here, it will be sufficient to say that acids which are well separated from each

Table 1. Lewis acid descriptors ${ }^{a}$ for a preliminary study

\begin{tabular}{|c|c|c|c|c|c|c|c|c|c|c|}
\hline Acids & 1 & 2 & 3 & 4 & 5 & 6 & 7 & 8 & 9 & 10 \\
\hline $1 \mathrm{AlCl}_{3}$ & 2.1 & 704.2 & 628.8 & 110.47 & 91.84 & 2.26 & 102 & 15.8 & 12.01 & - \\
\hline $2 \mathrm{BF}_{3}$ & 0 & 1137 & 1120.33 & 254.12 & 50.46 & 1.295 & 154 & 3.9 & 15.5 & - \\
\hline $3 \mathrm{MoS}_{2}$ & - & 235.1 & 225.9 & 62.59 & 63.55 & - & - & - & - & - \\
\hline $4 \mathrm{SnCl}_{4}$ & 0 & 511.3 & 440.1 & 258.6 & 165.3 & 2.43 & 7.6 & 2.87 & - & -115 \\
\hline $5 \mathrm{SO}_{2}$ & 1.63 & 320.5 & - & 237.6 & - & 1.4321 & 119 & 15.4 & 12.34 & -18.2 \\
\hline $6 \mathrm{POCl}_{3}$ & 2.4 & 519.1 & 520.8 & 222.46 & 138.78 & 1.95 & 122 & 13.3 & 11.89 & -67.8 \\
\hline $7 \mathrm{Me}_{3} \mathrm{~B}$ & - & 143.1 & 32.1 & 238.9 & - & 1.56 & 89 & - & 10.69 & - \\
\hline $8 \mathrm{Me}_{3} \mathrm{Al}$ & - & 136.4 & 99 & 209.41 & 155.6 & - & 61 & 2.9 & 9.76 & - \\
\hline $9 \mathrm{Me}_{2} \mathrm{SnCl}_{2}$ & 3.56 & 336.4 & - & - & - & 2.37 & - & - & 10.43 & - \\
\hline $10 \mathrm{TiO}_{2}$ & - & 913.4 & 853.9 & 56.3 & 9.96 & 1.97 & 160 & 48 & 10.2 & 0 \\
\hline $11 \mathrm{ZnCl}_{2}$ & 2.12 & 415.05 & 369.39 & 111.46 & 71.34 & 2.32 & 96 & - & 12.9 & -65 \\
\hline $12 \mathrm{TiCl}_{3}$ & - & 720.9 & 653.5 & 139.7 & 97.2 & 2.138 & 110 & - & - & 1110 \\
\hline $13 \mathrm{TiCl}_{4}$ & 0 & 804.2 & 737.2 & 252.3 & 145.2 & 2.19 & 181 & 2.8 & 11.76 & -54 \\
\hline $14 \mathrm{VCl}_{4}$ & 0 & 576.8 & 503.27 & 242.44 & - & 2.03 & 92 & - & - & 1130 \\
\hline $15 \mathrm{CrCl}_{2}$ & - & 395.2 & 356.1 & 114.5 & 71.1 & 2.12 & 91 & - & 9.97 & 6890 \\
\hline $16 \mathrm{MnCl}_{2}$ & - & 481.3 & 440.3 & 118.2 & 72.9 & 2.32 & 98.8 & - & 11.02 & 14350 \\
\hline $17 \mathrm{FeCl}_{2}$ & - & 341.79 & 302.3 & 117.95 & 76.65 & 2.38 & 95 & - & 10.34 & 14750 \\
\hline $18 \mathrm{FeCl}_{3}$ & 1.28 & 404.6 & 398.3 & 146.4 & 128 & 2.32 & 81 & - & - & - \\
\hline $19 \mathrm{CoCl}_{2}$ & - & 325.2 & 282.2 & 106.5 & 78.5 & 2.53 & 86 & - & 10.6 & 12660 \\
\hline $20 \mathrm{NiCl}_{2}$ & 3.32 & 305.332 & 259.032 & 97.65 & 71.67 & - & 87 & - & 11.23 & 6145 \\
\hline $21 \mathrm{CuCl}_{2}$ & - & 220.1 & 175.1 & 108.07 & 71.88 & - & 91.5 & - & - & 1080 \\
\hline $22 \mathrm{GaCl}_{3}$ & 0.85 & 524.17 & 454.36 & 172 & - & 2.208 & 78.7 & - & 11.96 & -63 \\
\hline $23 \mathrm{GeCl}_{4}$ & 0 & 543.4 & - & 347.15 & 29.21 & 2.1 & 81 & 2.43 & 11.68 & -72 \\
\hline $24 \mathrm{AsCl}_{3}$ & 1.53 & 335.24 & 294.7 & 233.2 & - & 2.16 & 70 & 1.59 & 11.7 & -79.9 \\
\hline $25 \mathrm{BCl}_{3}$ & 0.61 & 427.2 & 387.4 & 206.3 & 106.7 & 1.75 & 109 & 0 & 11.62 & -59.9 \\
\hline $26 \mathrm{SiCl}_{4}$ & 0 & 601.54 & 569.32 & 328.6 & 145.17 & 2.019 & 95.3 & 2.4 & 12.06 & -88.3 \\
\hline $27 \mathrm{SbCl}_{3}$ & 3.9 & 381.75 & 324.1 & 186 & 107.84 & 2.325 & 74 & 33 & 10.75 & -86.7 \\
\hline $28 \mathrm{PCl}_{3}$ & 7.8 & 314.7 & 272.3 & 217.1 & - & 1.95 & 78.5 & 3.43 & 9.91 & - \\
\hline
\end{tabular}

${ }^{a}$ The values were compiled from standard reference handbooks. The data may differ from values given in Table 3 which were compiled from much more complete background material. The calculations in the preliminary study were carried out using the data given in Table 1. Descriptors: 1, dipole moment (vapour phase) (D); 2 , negative of standard enthalpy of formation ( $\left.\mathrm{kJ} \mathrm{mol}{ }^{-1}\right) ; 3$, negative of standard Gibbs energy of formation ( $\left.\mathrm{kJ} \mathrm{mol}{ }^{-1}\right) ; 4$, standard entropy $\left(\mathrm{J} \mathrm{mol}^{-1} \mathrm{~K}^{-1}\right) ; 5$, heat capacity $\left(\mathrm{J} \mathrm{mol}^{-1} \mathrm{~K}^{-1}\right) ; 6$, mean bond length $(\AA) ; 7$, mean bond energy ( $\left.\mathrm{kcal} \mathrm{mol}^{-1}\right) ; 8$, dielectric constant; 9 , ionization potential $(\mathrm{eV}) ; 10$, magnetic and diamagnetic suspectibility $\left(10^{-6} \mathrm{cgs}\right)$. 


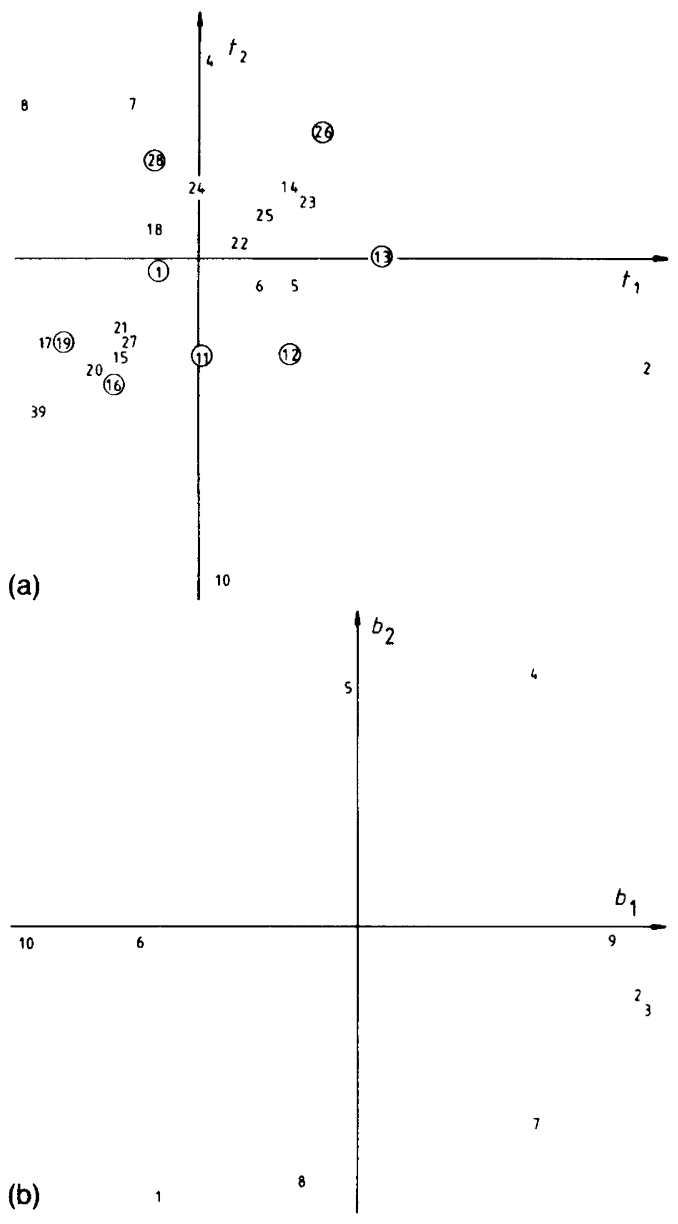

Fig. 1. (a) PC projections of descriptors in the preliminary study. (b) Loadings in the PC model. Loadings along the $b_{i}$ axis contribute to the $t_{i}$ component.

other and which have a broad distribution over the projection are obvious candidates for testing.

Experimental evaluation. A subset of 9 different Lewis acids was selected from the PC projection (encircled numbers in Fig. 1 (a)). The selected acids were used as catalysts in three reactions: $\mathrm{A}$, Reetz alkylation of silyl enol ethers ${ }^{4}$; B, Diels-Alder reaction; and C, Friedel-Crafts acylation. The specific reactions studied are shown in Scheme 1. The results obtained in these experiments are shown in Table 2. It was difficult to measure the initial rates of the reactions. As another, albeit rather rough, measure of the reactivity, we used $t_{510}$ which was defined as the time necessary to obtain $50 \%$ of the final yield.

Augmented study. The results of the initial study and the experimental evaluation were promising enough to justify an augmented study of Lewis acid descriptors, therefore, a set of 20 descriptors for 116 Lewis acids $\left(\mathrm{MX}_{n}, \mathrm{X}=\mathrm{F}, \mathrm{Cl}, \mathrm{Br}, \mathrm{I}\right)$ was compiled from various sources (see Table 3). A two-component PC model accounts for $65 \%$ of the variation in Table 3 . Inclusion of a third component did not improve the explained variance and was insignificant according to cross validation. The results of the PC analysis are shown in Figure 2.

\section{Strategies for selection}

The PC projections of the Lewis acids, Fig. 1 (a) and 2 (a)show the systematic variation in all the properties considered in Tables 1 and 3. We can use this information for a systematic search for test candidates. A subset of all the possible candidates can be selected in such a way that a suitable spread in all properties is assured. As with the selection of solvents discussed in a previous paper ${ }^{3}$, we can envisage several ways to proceed:

(1) Dissimilar acids: Select acids which are found on the periphery of the plot and placed far from each other. This will give a selection in which the dissimilarities are maximized.

(2) Uniform coverage: Select acids that are uniformly spread with regard to properties. This can

A:

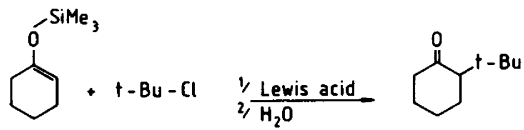

B:

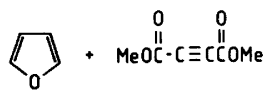

Lewis acid<smiles>COC(=O)C1C2C=CC(O2)C1C(=O)O</smiles><smiles>[CH2]CCc1ccccc1</smiles>

Lewis acid.

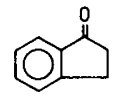

Scheme 1. Reactions used in screening experiments. 
be done by selecting candidates that form a regular lattice in the projection.

(3) Sequential simplex search: A sequential simplex strategy ${ }^{14}$ can be used to achieve a systematic search for a suitable catalyst. For this, the more complete projection in Fig. 2 (a) is preferable. The simplex search can be performed by selecting 3 acids near the center of the projection in such a way that their points form an approximately equilateral triangle in the projection. (If some acids are known to be useful, these are, of course, included in the initial simplex.) Run the experiments and determine the poorest candidate of the 3 , discard it and replace it with a new can-

(a)
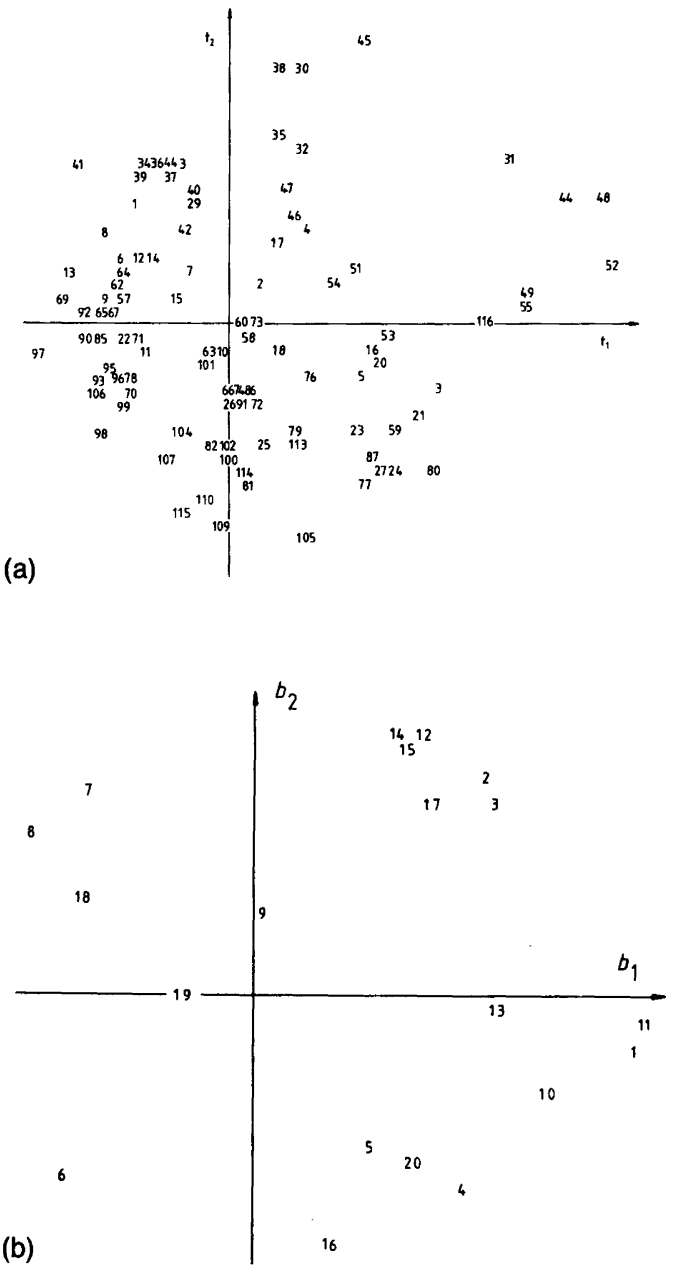

Table 2. Screening experiments with selected Lewis acids

\begin{tabular}{|c|c|c|c|}
\hline Reaction $^{a}$ & Lewis acid & $\begin{array}{l}\text { Maximum } \\
\text { yield (\%) }\end{array}$ & $\begin{array}{c}t_{50} \\
(\mathrm{~min})\end{array}$ \\
\hline A & $\begin{array}{l}\mathrm{AlCl}_{3} \\
\mathrm{CoCl}_{2} \\
\mathrm{MnCl}_{2} \\
\mathrm{PCl}_{3} \\
\mathrm{SiCl}_{4} \\
\mathrm{SnCl}_{4} \\
\mathrm{TiCl}_{3} \\
\mathrm{TiCl}_{4} \\
\mathrm{ZnCl}_{2}\end{array}$ & $\begin{array}{r}39 \\
0 \\
0 \\
0 \\
0 \\
38 \\
0 \\
45 \\
44\end{array}$ & $\begin{array}{c}1.9 \\
- \\
- \\
- \\
- \\
22.1 \\
- \\
3.1 \\
378\end{array}$ \\
\hline B & $\begin{array}{l}\mathrm{AlCl}_{3} \\
\mathrm{CoCl}_{2} \\
\mathrm{MnCl}_{2} \\
\mathrm{PCl}_{3} \\
\mathrm{SiCl}_{4} \\
\mathrm{SnCl}_{4} \\
\mathrm{TiCl}_{3} \\
\mathrm{TiCl}_{4} \\
\mathrm{ZnCl}_{2}\end{array}$ & $\begin{array}{c}50 \\
0 \\
0 \\
0 \\
0 \\
2.5 \\
13 \\
26 \\
0\end{array}$ & $\begin{array}{c}0.1 \\
- \\
- \\
- \\
- \\
20.2 \\
1.5 \\
7.0 \\
-\end{array}$ \\
\hline C & $\begin{array}{l}\mathrm{AlCl}_{3} \\
\mathrm{CoCl}_{2} \\
\mathrm{MnCl}_{2} \\
\mathrm{PCl}_{3} \\
\mathrm{SiCl}_{4} \\
\mathrm{SnCl}_{4} \\
\mathrm{TiCl}_{3} \\
\mathrm{TiCl}_{4} \\
\mathrm{ZnCl}_{2}\end{array}$ & $\begin{array}{c}94.1 \\
3.5 \\
6.6 \\
0 \\
0 \\
0 \\
25.7 \\
55.0 \\
5.0\end{array}$ & $\begin{array}{c}0.8 \\
630 \\
71.2 \\
- \\
- \\
- \\
36.0 \\
750 \\
1800\end{array}$ \\
\hline
\end{tabular}

a: Alkylation of silyl enol ether, B: Diels-Alder reaction, C: Friedel-Crafts reaction. See Scheme 1.

didate chosen so that the remaining 2 better candidates and the new 1 form a new simplex (triangle) oriented away from the first poorest candidate. Determine the worst outcome with the new simplex, etc. This allows for a systematic iterative search for a suitable acid catalyst.

These strategies are flexible and can take any prior information into account, such as details of the reaction mechanism. They can also cope with optimization by allowing for a systematic search around the winning candidate in a screening experiment so that various criteria of optimality

Fig. 2. (a) PC projection in the augmented study. (b) Loadings in the augmented study. 
such as yield, selectivity, ease of work-up, cost, etc. can be fulfilled.

\section{Discussion}

A selection strategy for screening experiments must enable chemically relevant test objects to be found. The strategies outlined above fulfill this criterion. The 3 reactions used to evaluate the initial study are all well established synthetic procedures. The 9 test candidates were selected based solely on their distribution in the PC projection, Fig. 1 (a). Boron trifluoride was an obvious candidate but was omitted due to the fact that it is a gas and difficult to dose. The results shown in Table 2 clearly demonstrate that the winning candidate in all 3 reactions is in complete agreement with established results: (A) titanium tetrachloride as the preferred catalyst in the $R e$ $e t z$ alkylation of silyl enol ethers ${ }^{4}$; (B) aluminum trichloride as a suitable catalyst for Diels-Alder reactions ${ }^{15}$; (C) aluminum trichloride as a preferred catalyst in Friedel-Crafts acylation. ${ }^{16}$ Iron(III) chloride has been reported to be a better catalyst than aluminum trichloride in some acylations ${ }^{17}$ so it is interesting to find that is very close to aluminum trichloride in the projections.

The PC projections also seem to contain information on the hard/soft properties of acids. ${ }^{2}$ In Fig. 1 (a) the $t_{1}$ axis seems to describe this property. The very hard acid $\mathrm{BF}_{3}$ is found on the extreme right in the projection and the soft acids $\mathrm{MoS}_{2}, \mathrm{CoCl}_{2}$ and $\mathrm{Me}_{3} \mathrm{~B}$ very far to the left. With the more complete data set in Table 3 (a), a slightly more complicated picture emerges and in the corresponding PC projection, Fig. 2 (a), the hard/soft properties are described by both components in a direction going from the lower left to the upper right quadrants. A tentative hard/soft scale could thus be defined as coordinates along an axis from the very hard acid, silicon tetrafluoride (48) to the very soft acid, copper(I) iodide (97).

A problem which is encountered whenever a set of descriptors is compiled for a large number of objects is that the data matrix cannot be completely filled. There are always missing data. Initially, about 35 Lewis acid descriptors were considered. However, some of them were available only for a handful of acids. In Table 3 , there are still data lacking for some of the acids. This does not pose any serious problems in the data analytic method used, PC analysis by the SIMCA program package, since this method tolerates a few $(<10 \%)$ missing data in some variables without serious loss of the overall information.

\section{Conclusions}

Chemistry of today suffers from a "data explosion" - any single chemical system can be characterized by a large number of measured properties; any single experiment can produce a multitude of measured responses. It is impossible to cope with the situation by mere inspection of tabulated data or by simple bivariate correlation. The only reasonable way to handle this is to use computer-assisted multivariate methods. The methods outlined in this paper and in Ref. 3 are examples of a general computer-assisted strategy for selecting test objects in synthetic screening. The strategy also allows for a systematic optimization of reaction strategies by sequential simplex search.

\section{Calculations and experimental}

The calculations were carried out on a Zampo (8bit) or a Toshiba T1500 (16-bit) microcomputer. PC modelling was accomplished by the SIMCA program package (SIMCA-3B version). The program is written in BASIC and versions for standard CP/M or IBM PC-compatible microcomputers are available from Sepanova $A B$, Östrandsvägen 14, S-122 43 Enskede, Sweden and from Principal Data Components, 2505 Shepherd Blvd., Columbia, MO 65201, USA.

Lewis acids used in the screening experiments were supplied by Aldrich or Sigma. Pro analysi quality was used. The acids were stored in a desiccator over Siccapent ${ }^{\circledR}$ (Merck) and all handling of the acids was carried out in an atmosphere of dry nitrogen.

GLC analyses were performed on a PYE UNICAM GCD with a flame ionization detector. HPLC analyses were achieved using a LDC high pressure liquid chromatograph equipped with a Constametric $^{\circledR}$ III pump and a Spectromonitor ${ }^{\circledR}$ III detector. Peak areas were used for quantification by the internal standard technique; a Milton Roy C-10 integrator was used. 
Table 3. Lewis acids and descriptors ${ }^{a}$ used in principal component analysis

\begin{tabular}{|c|c|c|c|c|c|c|c|c|c|c|}
\hline \multirow[t]{2}{*}{ Acids } & \multicolumn{10}{|c|}{ Descriptors } \\
\hline & 1 & 2 & 3 & 4 & 5 & 6 & 7 & 8 & 9 & 10 \\
\hline $1 \mathrm{TiCl}_{2}$ & 23.6 & 513.8 & 464.4 & 87.4 & 69.83 & 2.25 & 1308.5 & 475 & 3.13 & 585.62 \\
\hline $2 \mathrm{TiCl}_{3}$ & 51.3 & 720.9 & 653.5 & 139.7 & 97.15 & 2.3 & 440 & 660 & 2.64 & 1220.45 \\
\hline $3 \mathrm{TiCl}_{4}$ & 94.5 & 804.2 & 737.2 & 252.34 & 145.18 & 2.19 & -25 & 136.4 & 1.726 & 2220 \\
\hline $4 \mathrm{VCl}_{3}$ & 52.8 & 561 & - & - & - & - & - & - & 3 & 1296.51 \\
\hline $5 \mathrm{VCl}_{5}$ & - & 570.2 & - & 235.3 & - & 2.03 & -28 & 148.5 & 1.816 & 2374.9 \\
\hline $6 \mathrm{CrCl}_{2}$ & 24 & 395.4 & 356.1 & 115.3 & 71.2 & 2.09 & 814 & - & 2.878 & 634.73 \\
\hline $7 \mathrm{CrCl}_{3}$ & 54.3 & 556.5 & 486.2 & 123 & 91.8 & 2.38 & 1152 & 1300 & 2.76 & 1356 \\
\hline $8 \mathrm{MnCl}_{2}$ & 24 & 481.29 & 440.5 & 118.24 & 72.93 & 2.09 & 650 & 1190 & 2.977 & 602.2 \\
\hline $9 \mathrm{FeCl}_{2}$ & 25.2 & 341.79 & 302.3 & 117.95 & 76.65 & 2.38 & 677 & - & 3.16 & 657 \\
\hline $10 \mathrm{FeCl}_{3}$ & 54.5 & 399.49 & 334 & 142.3 & 96.65 & - & 306 & 315 & 2.898 & 1365.4 \\
\hline $11 \mathrm{CoCl}_{2}$ & 25.5 & 312.5 & 269.9 & 109.2 & 78.5 & 2.53 & 7.24 & 1049 & 3.356 & 679.78 \\
\hline $12 \mathrm{NiCl}_{2}$ & 26.2 & 305.33 & 259.03 & 97.65 & 71.67 & 1.82 & 1001 & 973 & 3.55 & 700.619 \\
\hline $13 \mathrm{CuCl}$ & 7.85 & 137.2 & 119.86 & 86.2 & 48.5 & 2.3407 & 429 & 1490 & 4.14 & 260.513 \\
\hline $14 \mathrm{CuCl}_{2}$ & 26.9 & 220.1 & 175.7 & 108.07 & 71.88 & 2.09 & 493 & 993 & 3.986 & 729.94 \\
\hline $15 \mathrm{ZnCl}_{2}$ & 26.8 & 415.05 & 369.398 & 111.46 & 71.34 & 2.05 & 283 & 732 & 2.91 & 665.1 \\
\hline $16 \mathrm{BCl}_{3}$ & - & 427.2 & 387.4 & 206.3 & 106.7 & 1.75 & -107.3 & 12.5 & 1.349 & 1785 \\
\hline $17 \mathrm{AlCl}_{3}$ & 55.6 & 704.2 & 628.8 & 110.47 & 91.84 & 2.06 & 190 & 182.7 & 2.44 & 1310.51 \\
\hline $18 \mathrm{GaCl}_{3}$ & 57.4 & 523.4 & 455.2 & 135.2 & - & 2.09 & 77.9 & 201.3 & 2.47 & 1433 \\
\hline $19 \mathrm{SiCl}_{2}$ & - & 162.4 & 176.32 & 282 & 51.33 & 2 & - & - & - & 676.46 \\
\hline $20 \mathrm{SiCl}_{4}$ & - & 657 & 617 & 330.6 & 90.2 & 2.091 & -70 & 57.57 & 1.483 & 2492.77 \\
\hline $21 \mathrm{GeCl}_{4}$ & 103.7 & 504.8 & - & 347.5 & - & 2.1 & -49.5 & 84 & 1.8443 & 2.488 .6 \\
\hline $22 \mathrm{SnCl}_{2}$ & 22.7 & 350 & 302.1 & 122.5 & - & 2.42 & 246 & 652 & 3.94 & 581.93 \\
\hline $23 \mathrm{SnCl}_{4}$ & - & 511.3 & 440.1 & 258.6 & 165.3 & 2.31 & -33 & 114.1 & 2.226 & 2227.7 \\
\hline $24 \mathrm{PCl}_{5}$ & - & 374.9 & 305 & 364.58 & 112.8 & 2.03 & 166.8 & 162 & 4.63 & 4159.3 \\
\hline $25 \mathrm{AsCl}_{3}$ & 55.9 & 305 & 259.4 & 216.3 & - & 2.161 & -8.5 & 130.2 & 2.163 & 1386.5 \\
\hline $26 \mathrm{SbCl}_{3}$ & 49.4 & 382.2 & 323.7 & 184.7 & 108 & 2.325 & 73.4 & 283 & 3.14 & 1231 \\
\hline $27 \mathrm{SbCl}_{5}$ & - & 440.2 & 350.2 & 301.3 & - & 1.97 & 2.8 & 79 & 2.336 & 3537 \\
\hline $28 \mathrm{AsCl}_{5}$ & - & - & - & - & - & - & - & - & - & 3990 \\
\hline $29 \mathrm{TiF}_{2}$ & 26.1 & 682.34 & 694.9 & 255.56 & 58.86 & 1.88 & - & - & - & 585.62 \\
\hline $30 \mathrm{TiF}_{3}$ & 56 & 1436.2 & 1360.7 & 87.9 & 92.02 & 1.97 & 1200 & 1400 & 3.4 & 1220.45 \\
\hline $31 \mathrm{TiF}_{4}$ & 101.6 & 1649.3 & 1559.3 & 133.97 & 114.2 & 1.92 & 400 & 284 & 2.798 & 2220 \\
\hline $32 V F_{3}$ & 57.6 & - & - & - & - & - & 800 & - & 3.363 & 1296.51 \\
\hline $33 \mathrm{VF}_{4}$ & - & 1404 & - & 121.4 & - & - & 325 & - & 2.975 & 2374.9 \\
\hline $34 \mathrm{CrF}_{2}$ & 21.1 & 779.9 & 711.3 & 89.7 & - & 1.72 & 894 & 1300 & 4.11 & 634.73 \\
\hline $35 \mathrm{CrF}_{3}$ & 58.1 & 1113.9 & 1046 & 93.95 & - & 1.9 & 1100 & 1200 & 3.8 & 1356 \\
\hline $36 \mathrm{MnF}_{2}$ & 25.7 & 795.5 & - & 92.26 & 66.78 & 1.724 & 856 & - & 3.98 & 602.2 \\
\hline $37 \mathrm{FeF}_{2}$ & 27.2 & 711.3 & 668.6 & 86.12 & 68.1 & 1.99 & 1000 & - & 4.09 & 657 \\
\hline $38 \mathrm{FeF}_{3}$ & 58.5 & 1046.4 & 840.9 & 98.4 & - & 1.92 & 1000 & - & 3.52 & 1365.4 \\
\hline $39 \mathrm{CoF}_{2}$ & 27.7 & 692.9 & 626.6 & 82.1 & 68.9 & 2.04 & 1200 & 1400 & 4.46 & 679.78 \\
\hline $40 \mathrm{NiF}_{2}$ & 28.5 & 651.4 & 604.1 & 73.6 & 64.06 & 1.72 & 1000 & - & 4.63 & 700.619 \\
\hline $41 \mathrm{CuF}$ & 8.61 & 192.5 & 171.6 & 64.8 & 44.9 & 1.749 & 908 & 1100 & - & 260.513 \\
\hline $42 \mathrm{CuF}_{2}$ & 28.9 & 542.7 & 475.4 & 86.12 & 94.14 & 1.72 & 950 & - & 4.23 & 729.94 \\
\hline $43 \mathrm{ZnF}_{2}$ & 28.8 & 764.4 & 713.3 & 73.68 & 65.65 & 1.81 & 872 & 1500 & 4.95 & 665.1 \\
\hline $44 \mathrm{BF}_{3}$ & - & 1137 & 1120.33 & 254.12 & 50.46 & 1.295 & -126.7 & -99.9 & 2.99 & 1785 \\
\hline $45 \mathrm{AlF}_{3}$ & 61.2 & 1504.1 & 1425 & 66.44 & 75.1 & 1.63 & 1291 & - & 2.882 & 1310.51 \\
\hline $46 \mathrm{GaF}_{3}$ & 61.5 & - & - & - & - & 1.88 & 800 & 1000 & 4.47 & 1433 \\
\hline $47 \mathrm{SiF}_{2}$ & - & 587.85 & 598.27 & 256.18 & 44.5 & 1.591 & - & - & - & 676.46 \\
\hline $48 \mathrm{SiF}_{4}$ & - & 1614.9 & 1572.7 & 282.4 & 73.6 & 1.55 & -90 & - & - & 2492.77 \\
\hline $49 \mathrm{GeF}_{4}$ & 108.7 & 1192.5 & - & 302.9 & - & 1.68 & -32 & -36.5 & 2.46 & 2488.6 \\
\hline $50 \mathrm{SnF}_{2}$ & 25 & - & - & - & - & 2.06 & - & - & - & 581.93 \\
\hline $51 \mathrm{SnF}_{4}$ & - & - & - & - & - & 1.86 & 705 & - & 4.78 & 2227.7 \\
\hline $52 \mathrm{PF}_{5}$ & - & 1595.8 & 1508.7 & 300.6 & 84.8 & 1.58 & -83 & -75 & 5.805 & 4152.3 \\
\hline
\end{tabular}


CARLSON, LUNDSTEDT, NORDAHL AND PROCHAZKA

Table 3. cont.

\begin{tabular}{|c|c|c|c|c|c|c|c|c|c|c|}
\hline \multirow[t]{2}{*}{ Acids } & \multicolumn{10}{|c|}{ Descriptors } \\
\hline & 11 & 12 & 13 & 14 & 15 & 16 & 17 & 18 & 19 & 20 \\
\hline $1 \mathrm{TiCl}_{2}$ & 2431 & 120 & - & - & - & - & - & 570 & - & 1.54 \\
\hline $2 \mathrm{TiCl}_{3}$ & 5134 & 111 & - & - & - & - & - & 1110 & - & - \\
\hline $3 \mathrm{TiCl}_{4}$ & 9431 & 104 & - & - & - & - & 11.76 & -54 & 0 & - \\
\hline $4 \mathrm{VCl}_{3}$ & 5322 & 101 & - & - & - & - & 15.8 & 3030 & - & - \\
\hline $5 \mathrm{VCl}_{5}$ & - & 91 & - & - & - & - & 11.77 & 113 & - & - \\
\hline $6 \mathrm{CrCl}_{2}$ & 2455 & 97 & - & - & - & - & 9.97 & 7230 & - & 1.66 \\
\hline $7 \mathrm{CrCl}_{3}$ & 5473 & 86 & - & - & - & - & - & 6890 & - & - \\
\hline $8 \mathrm{MnCl}_{2}$ & 2362 & 96 & - & - & - & - & 11.03 & 14350 & - & 1.55 \\
\hline $9 \mathrm{FeCl}_{2}$ & 2525 & 98 & - & - & - & - & 9.84 & 14750 & - & 1.83 \\
\hline $10 \mathrm{FeCl}_{3}$ & 5364 & 80 & - & - & - & - & - & 13450 & - & - \\
\hline $11 \mathrm{CoCl}_{2}$ & 2709 & 92 & - & - & - & - & 10 & 12.66 & - & 1.88 \\
\hline $12 \mathrm{NiCl}_{2}$ & 2753 & 90 & - & - & - & - & 11.23 & 6145 & - & 1.91 \\
\hline $13 \mathrm{CuCl}$ & 921 & 88 & 34.6 & 45.2 & .279 & -.279 & 10.7 & -40 & - & 1.9 \\
\hline $14 \mathrm{CuCl}_{2}$ & 2774 & 72 & - & - & .484 & -.242 & 12.89 & 1080 & - & - \\
\hline $15 \mathrm{ZnCl}_{2}$ & 2690 & 78 & 38.3 & 40.2 & .328 & -.16 & 12.9 & -65 & - & - \\
\hline $16 \mathrm{BCl}_{3}$ & - & 106.1 & 58.5 & 45.8 & .357 & -.119 & 11.62 & -59.9 & 0 & 2.04 \\
\hline $17 \mathrm{AlCl}_{3}$ & 5376 & 101.5 & 39.5 & 62.2 & .576 & -.192 & 12.01 & - & 1.97 & 1.61 \\
\hline $18 \mathrm{GaCl}_{3}$ & 5217 & 86.8 & 46 & 32.7 & .312 & -.104 & 11.96 & -63 & - & 1.81 \\
\hline $19 \mathrm{SiCl}_{2}$ & - & 101 & - & - & - & - & 10.93 & - & - & - \\
\hline $20 \mathrm{SiCl}_{4}$ & - & 95.6 & 48.3 & 45.5 & .44 & -.11 & 11.8 & -88.3 & 0 & 1.9 \\
\hline $21 \mathrm{GeCl}_{4}$ & - & 81.2 & - & - & .264 & -.066 & 11.68 & -72 & 0 & 2.01 \\
\hline $22 \mathrm{SnCl}_{2}$ & 2276 & 93 & - & - & - & -.16 & 7.3 & -69 & - & 1.65 \\
\hline $23 \mathrm{SnCl}_{4}$ & 8355 & 75.3 & 41.4 & 31.6 & .352 & -.088 & 12.13 & -115 & 0 & 1.96 \\
\hline $24 \mathrm{PCl}_{5}$ & - & 63 & - & - & - & -.06 & 10.7 & -67.8 & 0 & - \\
\hline $25 \mathrm{AsCl}_{3}$ & - & 73.8 & 48.2 & 18.7 & .183 & -.061 & 10.55 & -79.9 & 1.55 & 2.18 \\
\hline $26 \mathrm{SbCl}_{3}$ & 5032 & 75 & 43.3 & 28.1 & .294 & -.098 & 10.2 & -86.7 & 3.8 & 2.05 \\
\hline $27 \mathrm{SbCl}_{5}$ & - & 60.4 & - & - & - & - & - & -120 & - & - \\
\hline $28 \mathrm{AsCl}_{5}$ & - & - & - & - & - & - & - & - & - & - \\
\hline $29 \mathrm{TiF}_{2}$ & 2724 & - & - & - & - & - & - & - & - & 1.54 \\
\hline $30 \mathrm{TiF}_{3}$ & 5644 & 144 & - & - & - & - & - & 1300 & - & - \\
\hline $31 \mathrm{TiF}_{4}$ & 10012 & 142 & - & - & - & - & - & - & 0 & - \\
\hline $32 \mathrm{VF}_{3}$ & 5895 & 134 & - & - & - & - & - & 2730 & - & - \\
\hline $33 V F_{4}$ & - & - & - & - & - & - & - & - & - & - \\
\hline $34 \mathrm{CrF}_{2}$ & 2778 & 114 & - & - & - & - & 10.6 & - & - & 1.66 \\
\hline $35 \mathrm{CrF}_{3}$ & 5958 & 111 & - & - & - & - & - & 4370 & - & - \\
\hline $36 \mathrm{MnF}_{2}$ & 2644 & 111 & - & - & - & - & 11.38 & 10700 & - & 1.55 \\
\hline $37 \mathrm{FeF}_{2}$ & 2769 & 117 & - & - & - & - & - & 9500 & - & 1.83 \\
\hline $38 \mathrm{FeF}_{3}$ & 5870 & 110 & - & - & - & - & - & 13760 & - & - \\
\hline $39 \mathrm{CoF}_{2}$ & 2878 & 113 & - & - & - & - & - & 9490 & - & 1.88 \\
\hline $40 \mathrm{NiF}_{2}$ & 2845 & 112 & - & - & - & - & - & 2410 & - & 1.91 \\
\hline $41 \mathrm{CuF}$ & - & 102 & 26.1 & 69.8 & .366 & -.366 & - & - & - & 1.9 \\
\hline $42 \mathrm{CuF}_{2}$ & 3046 & 91 & - & - & - & - & - & 1050 & - & - \\
\hline $43 \mathrm{ZnF}_{2}$ & 2930 & 99 & 27.2 & 63.3 & .452 & -.226 & 13.91 & -38.2 & - & - \\
\hline $44 \mathrm{BF}_{3}$ & - & 154.3 & 71.5 & 86.7 & .504 & -.168 & 15.96 & - & 0 & 2.04 \\
\hline $45 \mathrm{AlF}_{3}$ & 5924 & 141 & 44.5 & 100.4 & .729 & -.243 & - & -13.4 & - & 1.61 \\
\hline $46 \mathrm{GaF}_{3}$ & 6205 & 114 & 48.5 & 53.3 & .456 & -.152 & - & - & - & 1.81 \\
\hline $47 \mathrm{SiF}_{2}$ & - & 141 & - & - & - & -.24 & 10.78 & - & 1.23 & - \\
\hline $48 \mathrm{SiF}_{4}$ & - & 142.6 & 58.7 & 80.8 & .6 & -.15 & 15.19 & - & 0 & 1.9 \\
\hline $49 \mathrm{GeF}_{4}$ & - & 112.5 & 59.8 & 51.2 & .416 & -.104 & 16.06 & -50 & 0 & 2.01 \\
\hline $50 \mathrm{SnF}_{2}$ & 2551 & 116 & - & - & - & - & - & - & - & 1.65 \\
\hline $51 \mathrm{SnF}_{4}$ & - & 101 & 47.8 & 59.6 & - & - & - & - & 0 & 1.96 \\
\hline $52 \mathrm{PF}_{5}$ & - & 111.1 & 50.6 & 60.5 & - & - & 15.54 & - & 0 & - \\
\hline
\end{tabular}


Table 3. cont.

\begin{tabular}{|c|c|c|c|c|c|c|c|c|c|c|}
\hline \multirow[t]{2}{*}{ Acids } & \multicolumn{10}{|c|}{ Descriptors } \\
\hline & 1 & 2 & 3 & 4 & 5 & 6 & 7 & 8 & 9 & 10 \\
\hline $53 \mathrm{AsF}_{3}$ & 61.5 & 821.3 & 774.16 & 181.2 & 126.57 & 1.712 & -8.5 & -63 & 2.666 & 1386.5 \\
\hline $54 \mathrm{SbF}_{3}$ & 53.7 & 915.5 & - & - & - & 1.9 & 292 & 319 & 4.379 & 1231 \\
\hline $55 \mathrm{SbF}_{5}$ & - & - & - & - & - & - & 7 & 149.5 & 2.99 & 3537 \\
\hline $56 \mathrm{AsF}_{5}$ & - & - & - & - & - & - & -80 & -53 & 7.71 & 3990 \\
\hline $57 \mathrm{TiBr}_{2}$ & 22.9 & 402 & 383.2 & 119.7 & 77.82 & 2.4 & 500 & 935.8 & 4.31 & 585.62 \\
\hline $58 \mathrm{TiBr}_{3}$ & 50 & 548.5 & 523.8 & 176.6 & 101.71 & 2.4 & - & 794.2 & - & 1220.45 \\
\hline $59 \mathrm{TiBr}_{4}$ & 92.9 & 616.7 & 589.5 & 243.5 & 131.5 & 2.31 & 39 & 230 & 2.6 & 2220 \\
\hline $60 \mathrm{VBr}_{3}$ & 51.8 & 447.9 & - & 142.4 & - & - & - & - & - & 1297.51 \\
\hline $61 \mathrm{VBr}_{4}$ & - & 393.6 & - & 334.9 & - & 2.3 & - & - & 4 & 2379 \\
\hline $62 \mathrm{CrBr}_{2}$ & 23.3 & 338.9 & - & - & - & 2.24 & 844 & - & 4.356 & 634.73 \\
\hline $63 \mathrm{CrBr}_{3}$ & 53.1 & 426.8 & - & - & - & 2.57 & 1130 & - & 4.29 & 1356 \\
\hline $64 \mathrm{MnBr}_{2}$ & 23.2 & 384.9 & - & 138.1 & - & 2.24 & - & - & 4.385 & 602.2 \\
\hline $65 \mathrm{FeBr}_{2}$ & 24.4 & 249.8 & 238.1 & 140.6 & 80.2 & 2.24 & 684 & - & 4.636 & 657 \\
\hline $66 \mathrm{FeBr}_{3}$ & 53.7 & 268.2 & - & 173.7 & - & - & - & - & - & 1365.4 \\
\hline $67 \mathrm{CoBr}_{2}$ & 24.9 & 221 & - & 134 & - & 2.24 & 844 & - & - & 679.78 \\
\hline $68 \mathrm{NiBr}_{2}$ & 25.5 & 212.11 & - & 136 & - & 2.24 & 963 & - & 5.098 & 700.619 \\
\hline $69 \mathrm{CuBr}$ & 7.78 & 104.6 & 100.8 & 96.11 & 54.73 & 2.173 & 492 & 1345 & 4.98 & 260.513 \\
\hline $70 \mathrm{CuBr}_{2}$ & 26.6 & 141.8 & - & 133.9 & - & 2.4597 & 498 & - & 4.77 & 729.94 \\
\hline $71 \mathrm{ZnBr}_{2}$ & 26.2 & 328.65 & 312.13 & 138.5 & - & 2.24 & 394 & 650 & 4.201 & 665.1 \\
\hline $72 \mathrm{BBr}_{3}$ & - & 239.7 & 238.5 & 229.7 & 128 & 1.87 & -46 & 91.3 & 2.6431 & 1785 \\
\hline $73 \mathrm{AlBr}_{3}$ & 54.3 & 527.2 & 504.4 & 180.2 & 101.7 & 2.21 & 97.5 & 263.3 & 2.64 & 1310.51 \\
\hline $74 \mathrm{GaBr}_{3}$ & 56.5 & 386.9 & - & 180 & - & 2.28 & 121.5 & 278.8 & 3.69 & 1433 \\
\hline $75 \mathrm{SiBr}_{2}$ & - & - & - & - & - & - & - & - & - & 676.46 \\
\hline $76 \mathrm{SiBr}_{4}$ & - & 92.13 & - & - & - & 2.15 & - & - & - & 2492.2 \\
\hline $77 \mathrm{GeBr}_{4}$ & 101.7 & 330.8 & - & 396.9 & - & 2.31 & 26.1 & 186.5 & 3.132 & 2488.6 \\
\hline $78 \mathrm{SnBr}_{2}$ & 22.1 & 264.8 & 248.9 & 146 & - & 2.55 & 215.5 & 620 & 5.117 & 581.93 \\
\hline $79 \mathrm{SnBr}_{4}$ & - & 377.4 & 350.2 & 264.4 & - & 2.44 & 31 & 202 & 3.34 & 2227.7 \\
\hline $80 \mathrm{PBr}_{5}$ & - & 269.9 & - & - & - & 2.1 & 100 & 106 & - & 4159.3 \\
\hline $81 \mathrm{AsBr}_{3}$ & 54.7 & 130 & 159 & 363.87 & 76.16 & 2.33 & 32.8 & 221 & 3.54 & 1386.5 \\
\hline $82 \mathrm{SbBr}_{3}$ & 48.4 & - & - & - & - & 2.51 & 96.6 & 280 & 4.148 & 1231 \\
\hline $83 \mathrm{SbBr}_{5}$ & - & - & - & - & - & - & - & - & - & 3537 \\
\hline $84 \mathrm{AsBr}_{5}$ & - & - & - & - & - & - & - & - & - & 3990 \\
\hline $85 \mathrm{Til}_{2}$ & 22 & 264 & 258.9 & 138.1 & 86.22 & 2.59 & 600 & 1000 & 4.99 & 585.62 \\
\hline $86 \mathrm{Til}_{3}$ & 48.9 & 322.2 & 318.47 & 192.5 & 116.8 & - & - & 727 & - & 1220.45 \\
\hline $87 \mathrm{Til}_{4}$ & 91.2 & 375.7 & 371.5 & 249.4 & 125.65 & - & 150 & 377.1 & 4.3 & 2220 \\
\hline $88 \mathrm{VI}_{3}$ & 50.7 & 280.5 & - & 203.1 & - & - & - & - & - & 1296.51 \\
\hline $89 \mathrm{VI}_{4}$ & - & - & - & - & - & - & - & - & - & 2374.9 \\
\hline $90 \mathrm{Crl}_{2}$ & 22.4 & 158.3 & - & - & - & 2.43 & 868 & 800 & 5.196 & 634.73 \\
\hline $91 \mathrm{Crl}_{3}$ & 51.9 & 205.1 & - & 199.6 & - & - & 600 & 350 & 4.915 & 1356 \\
\hline $92 \mathrm{MnI}_{2}$ & 22.5 & 331 & - & - & - & 2.43 & 638 & 500 & 5 & 602.2 \\
\hline $93 \mathrm{Fel}_{2}$ & 23.7 & 113 & 86.5 & 170 & 112.9 & 2.43 & 587 & 1093 & 5.315 & 657 \\
\hline $94 \mathrm{Fel}_{3}$ & 52.8 & 71 & - & - & - & - & - & - & - & 1365.4 \\
\hline $95 \mathrm{Col}_{2}$ & 24.1 & 87.9 & - & 153.2 & - & 2.43 & 515 & 570 & 5.68 & 679.78 \\
\hline $96 \mathrm{Nil}_{2}$ & 24.9 & 78.2 & - & 154 & - & 2.43 & 797 & - & 5.834 & 700.619 \\
\hline $97 \mathrm{Cul}$ & 7.65 & 67.8 & 69.5 & 96.7 & 54.06 & 2.6169 & 605 & 1290 & 5.62 & 260.513 \\
\hline $98 \mathrm{Cul}_{2}$ & 25.9 & 7.1 & - & - & - & 2.43 & - & - & - & 729.94 \\
\hline $99 \mathrm{ZnI}_{2}$ & 25.5 & 208.03 & 208.95 & 161.1 & - & 2.38 & 446 & 624 & 4.7363 & 665.1 \\
\hline $100 \mathrm{BI}_{3}$ & - & 71.13 & 20.72 & 349.18 & 70.79 & 2.1 & - & - & - & 1785 \\
\hline $101 \mathrm{AlI}_{3}$ & 52.9 & 313.8 & 300.8 & 159 & 98.7 & 2.53 & 191 & 360 & 3.98 & 1310.51 \\
\hline $102 \mathrm{Gal}_{3}$ & 55.4 & 239.4 & - & 203.9 & - & 2.44 & 212 & 345 & 4.15 & 1433 \\
\hline $103 \mathrm{Sil}_{2}$ & - & 144 & - & - & - & - & - & - & - & 676.46 \\
\hline $104 \mathrm{Sil}_{4}$ & - & 199 & - & 265.6 & - & 2.435 & - & - & - & - \\
\hline 35 Acta Ch & ica Scand & ica B $40(19$ & & & & & & & & 29 \\
\hline
\end{tabular}


CARLSON, LUNDSTEDT, NORDAHL AND PROCHAZKA

Table 3. cont.

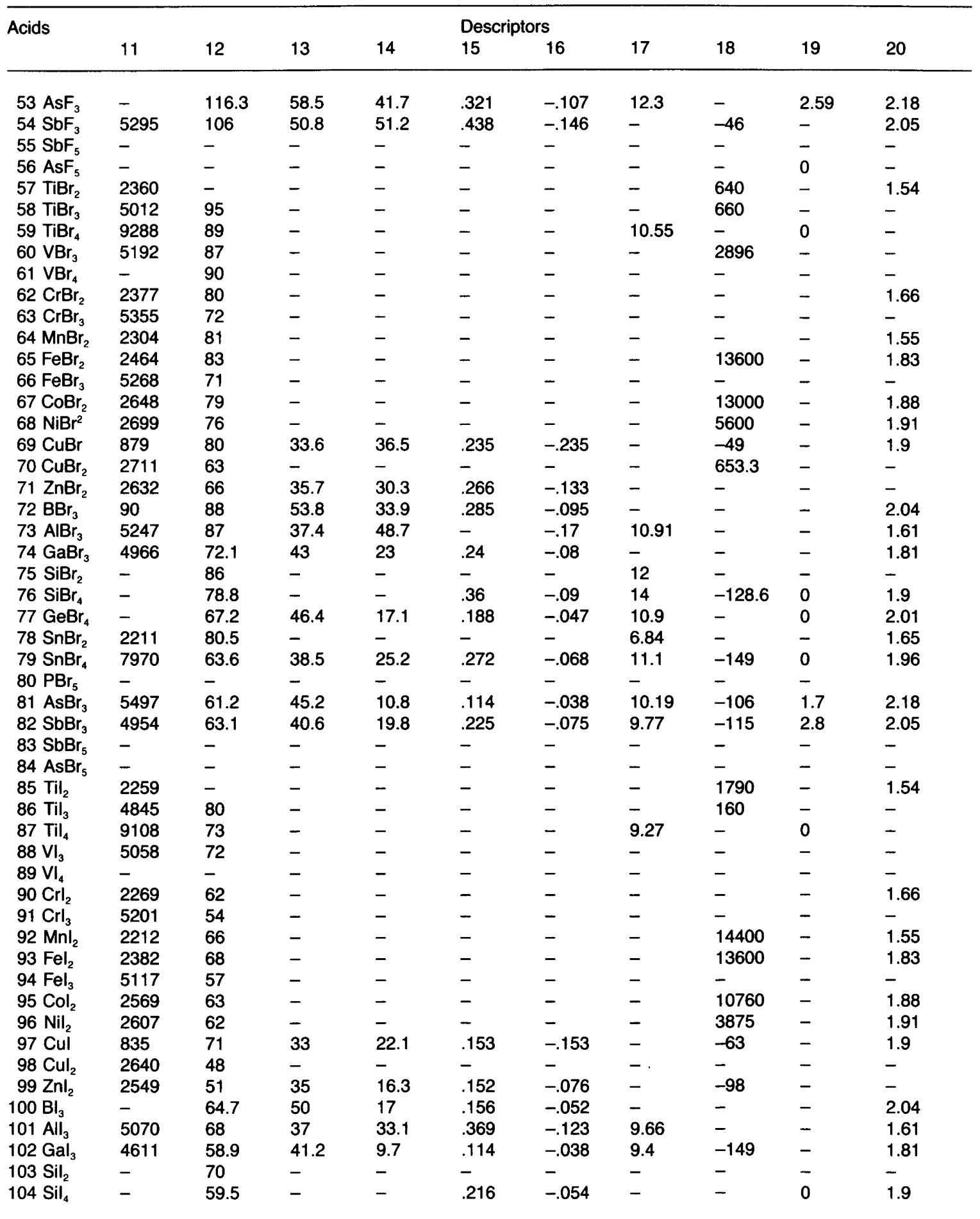


Table 3. cont

\begin{tabular}{|c|c|c|c|c|c|c|c|c|c|c|}
\hline \multirow[t]{2}{*}{ Acids } & \multirow[b]{2}{*}{1} & \multirow[b]{2}{*}{2} & \multirow[b]{2}{*}{3} & \multirow[b]{2}{*}{4} & \multicolumn{3}{|c|}{ Descriptors } & \multirow[b]{2}{*}{8} & \multirow[b]{2}{*}{9} & \multirow[b]{2}{*}{10} \\
\hline & & & & & 5 & 6 & 7 & & & \\
\hline $105 \mathrm{Gel}_{4}$ & 100.2 & 37.7 & - & 429.1 & - & 2.49 & 144 & 440 & 4.322 & 2488.6 \\
\hline $106 \mathrm{Snl}_{2}$ & 21.3 & 143.5 & - & 167.8 & - & 2.73 & 320 & 714 & 5.285 & 581.93 \\
\hline $107 \mathrm{Snl}_{4}$ & - & 143.9 & 143.9 & 168.6 & 84.9 & 2.67 & 144.2 & 364.5 & 4.473 & 2227.7 \\
\hline $108 \mathrm{PI}_{5}$ & - & - & - & - & - & - & - & - & - & 4159.3 \\
\hline $109 \mathrm{AsI}_{3}$ & 53.8 & - & - & - & - & 2.515 & 146 & 403 & 4.39 & 1386.5 \\
\hline $110 \mathrm{Sbl}_{3}$ & 47.3 & - & - & - & - & 2.67 & 170 & 401 & 4.917 & 1231 \\
\hline $111 \mathrm{Sbl}_{5}$ & - & - & - & - & - & - & 79 & 400.6 & - & 3537 \\
\hline $112 \mathrm{AsI}_{5}$ & - & - & - & - & - & - & 76 & - & 3.93 & 3990 \\
\hline $113 \mathrm{PCl}_{3}$ & - & 319.7 & 272.3 & 217.1 & - & 2.03 & -112 & 75.5 & 1.5751 & - \\
\hline $114 \mathrm{PBr}_{3}$ & - & 184.5 & 175.7 & 240.2 & - & 2.18 & -41.5 & 173.5 & 2.852 & - \\
\hline $115 \mathrm{PI}_{3}$ & - & 46.6 & - & - & - & 2.43 & 61.2 & 200 & 4.18 & - \\
\hline $116 \mathrm{PF}_{3}$ & - & 918.8 & 897.5 & 273.24 & 58.7 & 1.546 & -151.5 & -101.8 & 3.9 & - \\
\hline
\end{tabular}

${ }^{a}$ Compiled from standard reference tables (Refs. 5,6$)$ and other sources (Refs. 7-13). Descriptors: 1, coordinate bond energy (eV); 2 , negative of standard enthalpy of formation $\left(\mathrm{kJ} \mathrm{mol}^{-1}\right)$; 3 , negative of standard Gibbs energy of formation ( $\left.\mathrm{KJ} \mathrm{mol}^{-1}\right) ; 4$, standard entropy $\left(\mathrm{J} \mathrm{mol}^{-1} \mathrm{~K}^{-1}\right) ; 5$, heat capacity $\left(\mathrm{J} \mathrm{mol}^{-1} \mathrm{~K}^{-1}\right) ; 6$, mean bond length $(\AA) ; 7$, melting point $\left({ }^{\circ} \mathrm{C}\right) ; 8$, boiling point $\left({ }^{\circ} \mathrm{C}\right) ; 9$, density $\left(10^{3} \mathrm{~kg} \mathrm{~m}^{-3}\right) ; 10$, standard enthalpy of formation of $\mathrm{M}^{\mathrm{n}+}$ species $\left(\mathrm{kcal} \mathrm{mol}^{-1}\right)$;

\section{Procedures for screening experiments}

A: Reetz alkylation: A $20 \mathrm{ml}$ dichloromethane solution containing $1.70 \mathrm{~g}(10 \mathrm{mmol})$ of trimethylsilyloxycyclohexane, $0.93 \mathrm{~g}(10 \mathrm{mmol})$ of tert-butyl chloride, and an accurately weighed amount of nitrobenzene (internal standard) was maintained at $0^{\circ} \mathrm{C}$ by magnetic stirring. To this was added, in one portion a cooled $\left(0^{\circ} \mathrm{C}\right)$ solution of $10 \mathrm{mmol}$ of the Lewis acid in $20 \mathrm{ml}$ of dichloromethane. The reaction mixture was maintained at $0^{\circ} \mathrm{C}$ by means of an ice bath.

Analysis: Aliquots $(0.5 \mathrm{ml})$ were withdrawn at regular intervals and diluted with $5 \mathrm{ml}$ of dichloromethane. The sample was shaken with 10 $\mathrm{ml}$ of aqueous $8 \% \mathrm{NaHCO}_{3}$ until the organic layer became clear. This was analyzed by GLC using a $1.5 \mathrm{~m} \times 4 \mathrm{~mm}$ (i.d.) glass column packed with $5 \%$ PEG $+0.5 \% \mathrm{KOH}$ on Chromosorb ${ }^{\circledR}$ W-AW 100-120 mesh.

B: Diels-Adler reaction: A $20 \mathrm{ml}$ dichloromethane solution containing $1.42 \mathrm{~g} \mathrm{(10} \mathrm{mmol})$ of dimethyl acetylenedicarboxylate and an accurately weighed amount of nitrobenzene (internal standard) was agitated magnetically while a solution of $10 \mathrm{mmol}$ of the Lewis acid in $20 \mathrm{ml}$ of dichloromethane was added. The resulting mixture was stirred in a thermostated water bath at $22^{\circ} \mathrm{C}$. The reaction was started by the rapid addition of 0.68 $\mathrm{g}(10 \mathrm{mmol})$ of furan in $10 \mathrm{ml}$ of dichloromethane. The reaction mixture was stirred at $22^{\circ} \mathrm{C}$ and samples were withdrawn at regular intervals. Analysis: 5 drops of the reaction mixture were diluted with $5 \mathrm{ml}$ of methanol and $0.5 \mathrm{ml}$ of this solution was further diluted with $5 \mathrm{ml}$ of a methanol/water mixture, $38 / 62(\mathrm{v} / \mathrm{v})$. This very dilute solution was subjected to isocratic HPLC analysis using the same methanol/water mixture as eluent and a Hypersil ${ }^{\circledR} \mathrm{C}-18$ column. Peaks were detected by UV absorption at $289 \mathrm{~nm}$.

C: Friedel-Crafts acylation: A $20 \mathrm{ml}$ dichloromethane solution containing $10 \mathrm{mmol}$ of the Lewis acid was refluxed with magnetic stirring while $10 \mathrm{ml}$ of the reactant solution was rapidly added. This solution contained $1.69 \mathrm{~g}(10 \mathrm{mmol})$ of 3phenyl propanyl chloride and an accurately weighed amount of phenyl cyclohexane (internal standard). The mixture was maintained at reflux, samples $(\sim 0.5 \mathrm{ml})$ withdrawn at regular intervals 
Table 3. cont.

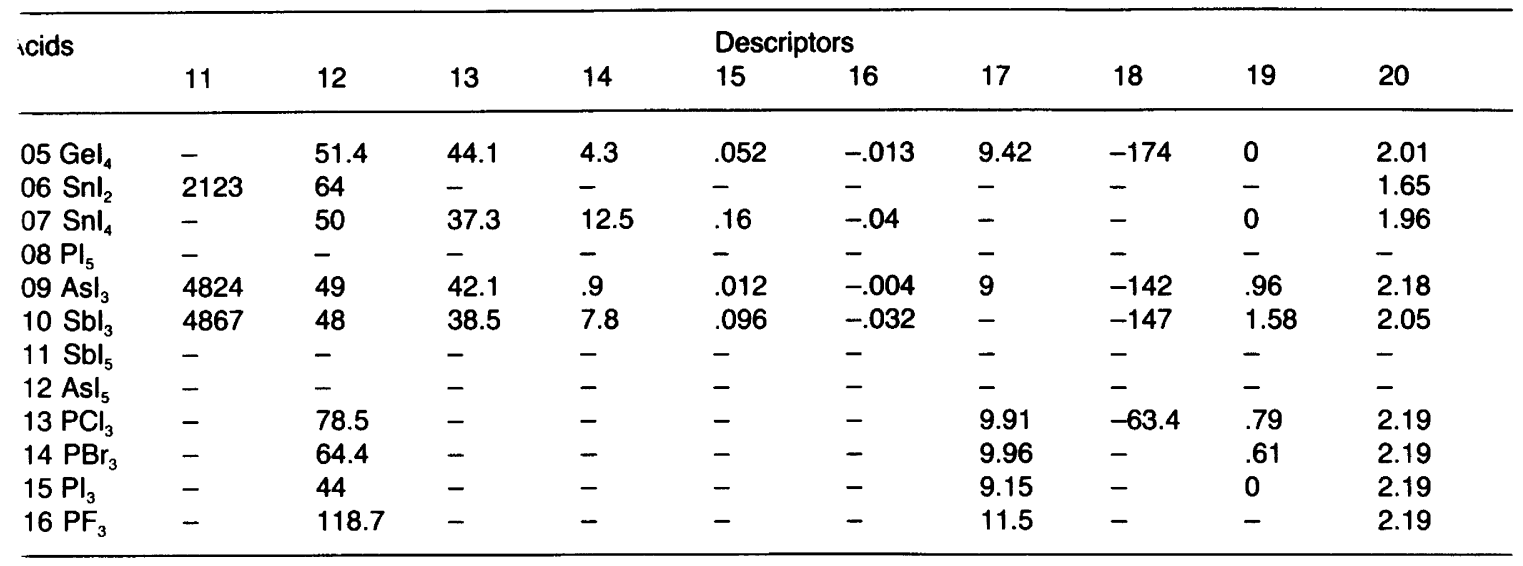

1, lattice energy (exp. or calc.) $\left(\mathrm{kJ} \mathrm{mol}^{-1}\right) ; 12$, mean bond energy (kcal mol-1); 13, covalent bond energy (kcal mol ${ }^{-1} ; 14$, unic bond energy ( $\left.\mathrm{kcal} \mathrm{mol}^{-1}\right) ; 15$, partial charge on central atom (e); 16, partial charge on ligand atom (e); 17, ionization otential (gas phase) $(\mathrm{eV}) ; 18$, magnetic susceptibility (measured at various temperatures $\left(10^{-6} \mathrm{cgs}\right)$ ); 19 , dipole moment jas phase) (D); 20 , atomic electronegativity of central atom in different oxidation states.

were shaken with $5 \mathrm{ml}$ of acidulated water and analyzed by GLC. A $1.5 \mathrm{~m} \times 4 \mathrm{~mm}$ i.d. glass column packed with $6 \%$ QF-1 on Chromosorb ${ }^{\circledR}$ W-AW, 100-120 mesh, was used.

Acknowledgements. Financial support from the Swedish Natural Science Research Council (NFR) and from the National Swedish Board for Technical Development (STU) is gratefully acknowledged.

We also express our gratitude to Profs. S. Wold and $\mathrm{E}$. Rosen for encouraging discussions and to Mr. S. Swanson for linguistic revision.

\section{References}

1. Jensen, W. B. The Lewis acid-base concepts. Wiley, New York, Chichester, Brisbane, Toronto, 1980.

2. Ho, T.-L. Hard and soft acids and bases principle in organic chemistry. Academic Press, New York, San Francisco, London 1977.

3. Carlson, R., Lundstedt, T. and Albano, C. Acta Chem. Scand. B 39 (1985) 79.

4. Reetz, M., Maier, W. F., Heimbach, H., Giannis, A. and Anastassious, G. Chem. Ber. 113 (1980) 3734.
5. (a) Handbook of chemistry and physics, 57th edition. CRC Press Inc., Cleveland, Ohio 1977;

(b) Cotrell, T. L. Strengths of chemical bonds, 2nd

Ed. Butterworths, London 1958;

(c) Lewin, R. D. and Lias, S. D. Ionization potentials and appearance potential measurements (19711981). Natl. Stand. Ref. Data Ser., Natl. Bur. of Stand. (US) 1982;

(d) Galasso, F. S. Structures and properties of inorganic compounds. Pergamon Press, Oxford, New York, Toronto, Sidney, Braunschweig, 1970.

6. (a) Pearson, R. G. and Mawly, R.J. Halogen chemistry, Vol. 3. Academic Press, London, New York 1967, pp. 55-85;

(b) International tables for $X$-ray crystallography. Kenoch Press, Birmingham, U.K. 1968;

(c) Sutton, L.E. (ed.) Tables of interatomic distances and configuration in molecules and ions. Chemical Society, London 1958, Supplement 1965; (d) Wagmann, D. D., Evans, W. H., Parker, V. B., Schumm, R. H., Churney, K. L. and Nuttal, R. L. J. Phys. Chem. Ref. Data 11, Supplement II (1982); (e) Kaye, T. H. and Laby, T. H. Tables of physical and chemical constants and some mathematical functions. Longman Group, Ltd., London 1973;

(f) Brandes, E. A. (ed.). Metal reference book, 6th Ed. Butterworths, London 1983;

(g) Sanderson, R. T. Chemical bonds and energy. Academic Press, San Francisco, London 1976;

(h) Nelson, Jr., R. D., Lide, D. R. and Mayott, 
A. A. Selected values of electric dipole moments in the gas phase. Natl. Stand. Ref. Data Ser., Natl. Bur. of Stand. (US) 10 1967;

(i) McClellan, A. L. Tables of experimental dipole moments. Freeman, San Fransisco 1963;

(j) Stull, D. R. and Prophet, H. J.A.N.A.F. Thermochemical Tables, Natl. Stand. Ref. Data Ser. 37. Natl. Bur. of Stand. (US) 1971.

7. Brewer, L., Somyajulu, G. R. and Brackett, J. Chem. Rev. 63 (1963) 111.

8. Sanderson, R. T. Polar covalence. Academic Press, New York, London, Paris, San Diego, Sao Paolo, Sydney, Toronto, 1983.

9. Jolly, W. L. Chem. Phys. Lett. 100 (1983) 546.

10. Mason, C. F. V. and Behrens, R. G. J. Less Common Met. 55 (1982) 21.

11. Moody, G.J. and Thomas, J.D.R. Dipole moments in inorganic chemistry. Edward Arnold, London, 1971.
12. Smith, J.W. Electric dipole moments. Butterworths, London, 1965.

13. Gray, H. B. Electrons and chemical bonding. Benjamin, New York, Amsterdam, 1965.

14. Spendley, H. G. R., Hext, G. R. and Himsworth, F. R. Technometrics 4 (1962) 441.

15. McCulloch, A.W., Smith, D. G. and McInnes, A. G. Can. J. Chem. 51 (1973) 4125.

16. Martin, H. L. and Fieser, L. F. Org. Synth. Coll. Vol. 2 (1943) 569.

17. (a) Gallo, R., Desbois, M. and Simeoni, M. Fourth European Symposium on Organic Chemistry. Aixen-Provence, September 2-6, 1985;

(b) Scuotto, J.-F., Mathieu, D., Gallo, R., PhanTan-Luu, R., Metzger, J. and Desbois, M. Bull. Soc. Chim. Belg. 94 (1985) 897.

Received December 4, 1985 